\title{
Permanent Pacemaker for Sick Sinus Syndrome with Absent Right Superior Vena Cava - A Case Report.
}

\author{
PK Chanda ${ }^{1}$, N Ahmed ${ }^{2}$, S Biswas ${ }^{1}$, MM Mehedi ${ }^{1}$, S DasGupta ${ }^{1}$, MA Rahman ${ }^{3}$, F Ahmed ${ }^{1}$ \\ ${ }^{1}$ Dept. of Cardiac Surgery, National Heart Foundation Hospital \& Research Institute, ${ }^{2}$ Dept. of \\ Cardiology, National Heart Foundation Hospital \& Research Institute, ${ }^{3}$ Cardiac Anesthesia, National \\ Heart Foundation Hospital \& Research Institute
}

\begin{abstract}
:
Key Words: A 42 year old patient, admitted into our hospital for permanent implantation. Implantation of a Permanent permanent pacemaker was attempted by the transvenous approach via venous puncture of cephalic pacemaker, superior vena cava, persistent left superior vena cava. vein \& subclavian vein on both sides but guide wire failed to pass. Coronary angiogram with cardiac catheterization was done \& revealed absence of right superior vena cava and presence of persistant left superior vena cava. Under endotracheal general anesthesia (GA) with limited left thoracotomy epicardial pacemaker was implanted.
\end{abstract}

(Cardiovasc. j. 2011; 3(2): 230-232)

\section{Introduction:}

Implantation of a permanent pacemaker (PPM) is mostly performed by puncturing cephalic vein or subclavian vein and introducing the pacemaker leads through the brachiocephalic vein, superior vena cava and then to the right atrium or right ventricle. Occasionally, abnormal venous anomaly will make the procedure unsuccessful. Such anomalies include the absence of right superior vena cava (SVC), presence of persistant left superior vena cava (PLSVC), absence of left brachiochephalic vein. It is very rare to implant the pacemaker facing absence of right SVC with presence of PLSVC. Here in, we report a case of absence of right SVC with presence of PLSVC. Here in, we reported a case of absence of right SVC with presence of PLSVC that was incidentally diagnosed during the implantation of a permanent pacemaker.

\section{Case Note:}

A 42 year old patient, admitted into our hospital on 13/05/2008 with the complaints of dizziness, palpitation and restlessness for the last 8 months and a single episode of syncopal attack very recently. He is normotensive, nondiabetic but smoker. On physical examination on admission pulse rate was found 40 beats/min. and did not demonstrate any other abnormality. Hence adjunct to his ECG findings he was diagnosed as a case of sick sinus syndrome. Transthoracic Echocardiography showed mild concentric hypertrophy, dilated coronary sinus with adequate LV systolic performance and were otherwise nonrevealing. Chest X-ray showed normal cardiac silhouethe without evidence of pulmonary congestion. Blood chemistries were within normal limits.

Under the diagnosis of tachycardia- bradycardia syndrome the patient underwent permanent pacemaker implantation on $14 / 05 / 08$. During the procedure venous access was attempted by via venous puncture of left cephalic vein and left subclavian vein first. However the guide wire failed to pass the SVC after several attempts. Similar attempts were also attempted on the right side but failed. On the very next day, his Coronary Angiogram with Cardiac Catheterization were done

Address for Correspondence: Dr. Prasanta Kumar Chanda, Associate Professor Cum Senior Consultant, Cardiac Surgery, National Heart Foundation Hospital \& Research Institute, Dhaka, Bangladesh. e-mail: pkchanda@gmail.com 
and revealed absence of SVC and presence of persistent PLSVC.

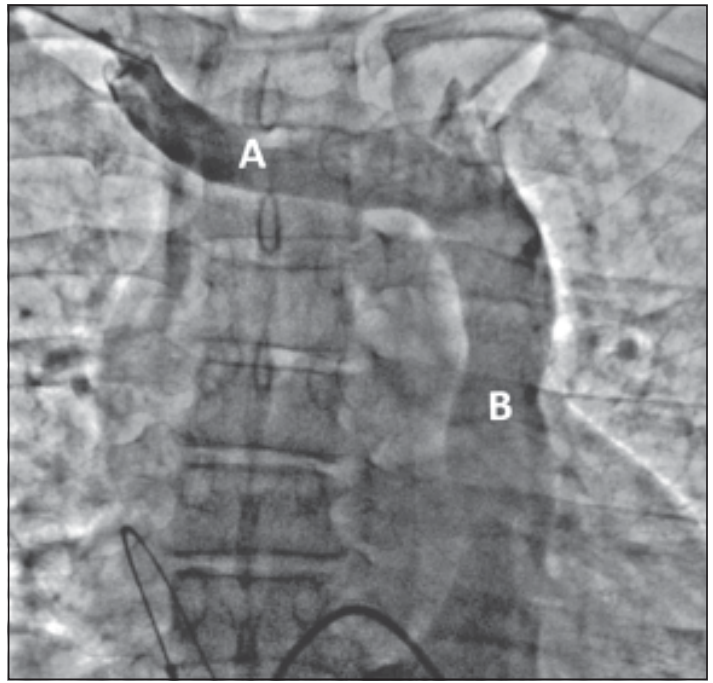

Fig.-1: Injection of contrast showing: (A) Rt. Brachiocephalic vein, draining into (B) sole left superior vena cava

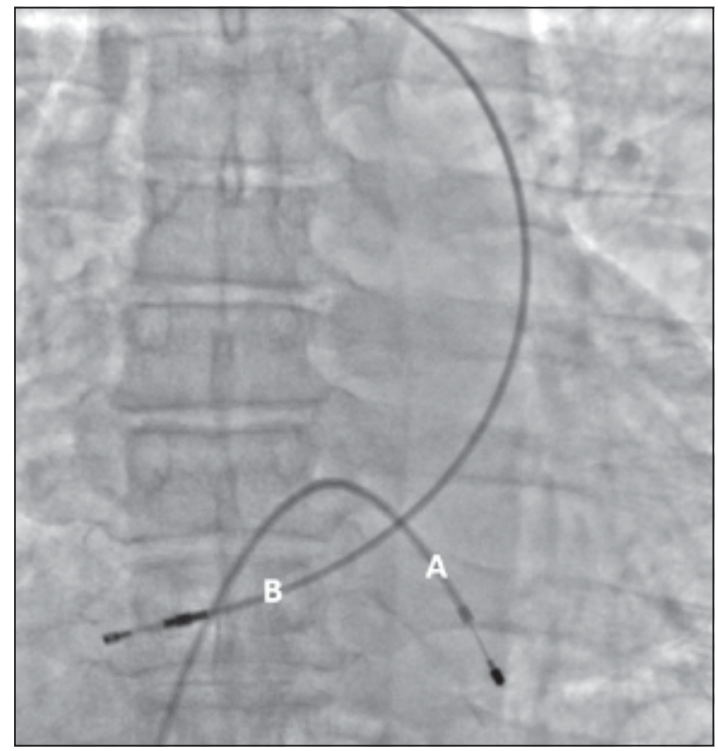

Fig.-2 (A) TPM in situ; (B) PPM lead failed to be inserted

On 18/05/08 under GA, with all aseptic precautions limited left anterolateral thoracotomy followed by pericardiotomy was done. An epicardial pacemaker lead was implanted on anterolateral wall of left ventricle. After checking pacing and haemostasis, wound was closed in layers leaving an intrathoracic tube. Post operatively the patient has been doing well with more than 2 years follow up.

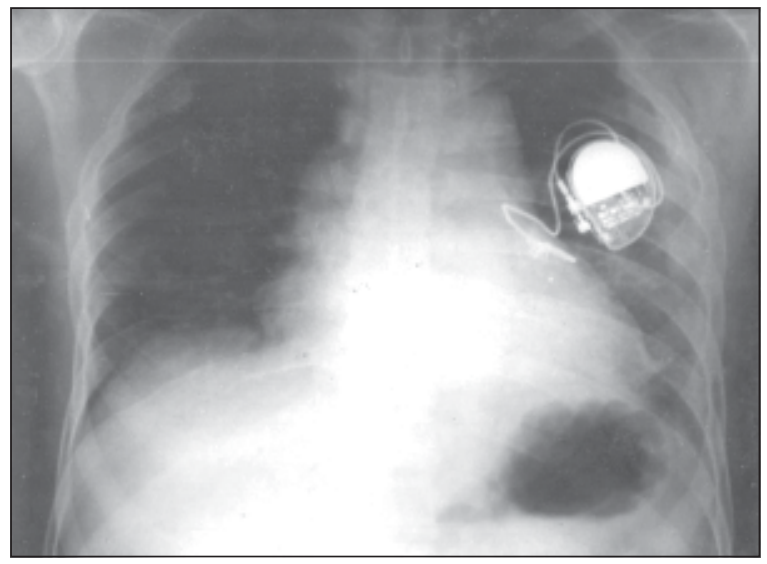

Fig.-3: Post implantation X-Ray showing epicardial PPM lead with generator.

\section{Discussion:}

A persistent left sided SVC is the commonest anomaly of the major veins. It occurs in 0.1 to $0.2 \%$ of the general population and between 3 and $8 \%$ in those with congenital cardiac malformations. ${ }^{1}$ An associated absent right SVC is much less common and fewer than 150 cases in patients with situs solitus have been reported. It is most likely to result from flow reversal between the left and right anterior cardinal and innominate veins during early development but the cause is not known. ${ }^{2}$

Associations with conduction system abnormalities also remain unclear. Fragmentation and stretching of the atrioventricular node over a large coronary sinus have been reported. ${ }^{3}$ The slow atrioventricular nodal pathway is usually situated close to the coronary sinus os. Presumably the very between the fast and slow pathways and this made selective ablation of one pathway difficult. Variation in the venous drainage system is not usually physiologically important and is probably often unrecognized during life.

\section{Conclusion:}

Implantation of a permanent pacemaker by the transvenous approach might fail from the absence of right superior vena cava (SVC) with presence of persistent left superior vena cava (PLSVC). The 
circuitous path taken by the pacemaker lead here explains the difficulty of transvenously obtaining a stable electrode position and sustained capture. Primary elective epicardial electrode implantation has been recommended for those with a persistent left sided SVC in association with an absent right SVC requiring pacemaker insertion. ${ }^{4}$

\section{References:}

1. Nsah EN, Moore GW, Hutchins GM. Pathogenesis of persistent left superior vena cava with a coronary sinus connection. Pediatr Pathol 1991; 11: 261-9.
2. Mooney DP, Snyder CL, Holder TM. An absent right and persistent left superior vena cava in an infant requiring extracorporeal membrane oxygenation therapy. F Pediatr Surg 1993; 28:1633-4.

3. James TN, Marshall TK, Edwards JE: Cardiac electrical instability in the presence of a left superior vena cava. Circulation 1976; 54:689-97.

4. Lenox CC, Zuberbuhler JR, Park SC, Neches WH, Mathews RA, Fricker FJ, et al. Absent right superior vena cava with persistent left superior vena cava : implications and management. Am F Cardiol 1980;45: 117-22. 